\title{
New var reconstruction algorithm exposes high var sequence diversity in a single geographic location in Mali
}

\author{
Antoine Dara ${ }^{1 \dagger}$, Elliott F. Drábek ${ }^{2+}$, Mark A. Travassos ${ }^{1}$, Kara A. Moser ${ }^{2}$, Arthur L. Delcher ${ }^{2}$, Qi Su², \\ Timothy Hostelley ${ }^{2}$, Drissa Coulibaly ${ }^{3}$, Modibo Daou $^{3^{\wedge}}$, Ahmadou Dembele $^{3}$, Issa Diarra ${ }^{3}$, Abdoulaye K. Kone $^{3}$, \\ Bourema Kouriba ${ }^{3}$, Matthew B. Laurens ${ }^{1}$, Amadou Niangaly ${ }^{3}$, Karim Traore ${ }^{3}$, Youssouf Tolo ${ }^{3}$, Claire M. Fraser ${ }^{2,4,5}$, \\ Mahamadou A. Thera ${ }^{3}$, Abdoulaye A. Djimde ${ }^{3}$, Ogobara K. Doumbo ${ }^{3}$, Christopher V. Plowe ${ }^{1}$ and Joana C. Silva ${ }^{2,4^{*}}$
}

\begin{abstract}
Background: Encoded by the var gene family, highly variable Plasmodium falciparum erythrocyte membrane protein-1 (PFEMP1) proteins mediate tissue-specific cytoadherence of infected erythrocytes, resulting in immune evasion and severe malaria disease. Sequencing and assembling the 40-60 var gene complement for individual infections has been notoriously difficult, impeding molecular epidemiological studies and the assessment of particular var elements as subunit vaccine candidates.

Methods: We developed and validated a novel algorithm, Exon-Targeted Hybrid Assembly (ETHA), to perform targeted assembly of var gene sequences, based on a combination of Pacific Biosciences and Illumina data.

Results: Using ETHA, we characterized the repertoire of var genes in 12 samples from uncomplicated malaria infections in children from a single Malian village and showed them to be as genetically diverse as vars from isolates from around the globe. The gene var2csa, a member of the var family associated with placental malaria pathogenesis, was present in each genome, as were vars previously associated with severe malaria.

Conclusion: ETHA, a tool to discover novel var sequences from clinical samples, will aid the understanding of malaria pathogenesis and inform the design of malaria vaccines based on PfEMP1.

ETHA is available at: https://sourceforge.net/projects/etha/.
\end{abstract}

Keywords: Malaria, Plasmodium falciparum, Plasmodium falciparum erythrocyte membrane protein-1, PfEMP1, var, var2csa, Mali, ETHA

\section{Background}

Naturally acquired immunity to malaria appears to occur, at least in part, through the acquisition of antibodies to parasite antigens expressed on the surface of infected erythrocytes. In epidemiological studies, having antibodies to these parasite-produced erythrocyte surface antigens is consistently associated with protection

\footnotetext{
*Correspondence: jcsilva@som.umaryland.edu

${ }^{\dagger}$ Equal contributors

Deceased

${ }^{2}$ Institute for Genome Sciences, University of Maryland School of Medicine, Baltimore, MD, USA

${ }^{4}$ Department of Microbiology and Immunology, University of Maryland

School of Medicine, Baltimore, MD, USA

Full list of author information is available at the end of the article
}

against clinical malaria $[1,2]$. The best studied of these variant surface antigen (VSA) families, Plasmodium falciparum erythrocyte membrane protein-1 (PfEMP1) antigens, are large molecules expressed on the surface of the infected erythrocyte $[3,4]$ that bind to endothelial receptors [5-8]. Each PfEMP1 typically contains between two and eight Duffy-binding-like (DBL) domains and one to two cysteine-rich interdomain regions (CIDR). PfEMP1s are encoded by the var family of genes, 40 to 61 of which have been found in the few $P$. falciparum assembled genomes available to date. An infected erythrocyte expresses only one PfEMP1 variant on its surface [9]. P. falciparum parasites undergo clonal antigenic variation that is central to their ability to evade 
the host immune response [10]. PfEMP1s mediate tissue-specific cytoadherence, which allows infected erythrocytes to sequester in small capillaries of targeted host tissue and evade clearance by the spleen, thereby producing devastating pathologic effects [11]. Compared to other $P$. falciparum genes, most var genes exhibit extreme diversity, with less than $50 \%$ shared amino acid sequence identity [12]. Such diversity has proven a major obstacle to the development of strategies to amplify and sequence new var genes from clinical samples, hampering the understanding of var gene biology and the role of PfEMP1 in clinical disease. The full complement of var genes is only known for a few reference genomes and two clinical samples [13]. Until now, it has thus not been possible to sequence vars with adequate completeness and from a number of samples sufficient to associate specific var elements with clinical outcomes such as pregnancy-associated or severe malaria [14].

The best characterized PfEMP1, VAR2CSA, is expressed on the surface of infected erythrocytes that bind to chondroitin sulfate in the placental matrix $[15,16]$. Antibodies to this antigen prevent placental cytoadherence and are associated with protection from placental malaria [15]. From the limited number of variants sequenced, VAR2CSA exhibits significantly greater sequence conservation than other vars, with over $75 \%$ shared amino acid identity among orthologs $[12,17,18]$, making it a promising subunit vaccine candidate against pregnancy-associated malaria. Better characterization of VAR2CSA diversity within and across different geographic locations would enable an informed assessment of the need for regionally-based vaccines versus a globally effective vaccine approach.

The study of rapidly evolving genes such as those that encode most variant surface antigen families relies on de novo genome assemblies and/or gene-targeted sequencing approaches. Assessment of genetic variation based on mapping of re-sequencing reads requires reads to map uniquely between isolates within a maximum sequence divergence cutoff (usually $\sim 2 \%$, or a maximum of two to three single nucleotide polymorphisms, SNPs, in a $100 \mathrm{bp}$ read). A large proportion of reads originating from var loci often cannot be reliably mapped across isolates, either because they map to repetitive regions present in multiple copies in the genome or because they differ among orthologous copies by more than the threshold cutoff necessary for mapping [19]. The long sequence reads generated with Pacific Biosciences (PacBio) technology have great utility as they allow the placement of repetitive regions in an assembly, using flanking unique conserved sequences as a genomic anchor $[20,21]$. However, if the PacBio reads are short in comparison to the length of VSA genes, the high error rate of PacBio-generated sequences, which can top 15\%, may lead to assembly errors, including chimeras among members of multigene families. In contrast, Illumina sequencing technologies produce much more accurate data, but the short read length poses a challenge for var gene assembly.

Here, we describe a novel, specialized pipeline that leverages publicly available var gene sequences and capitalizes on a combination of relatively low amounts of PacBio and Illumina data to reconstruct var gene sequences present in clinical malaria field samples. We show that all $P$. falciparum genomes from uncomplicated malaria infections harbor var2csa and also var gene subclasses previously associated with severe malaria. We also show a lack of clustering of VAR2CSA by geography and identify segments conserved across most var2csa sequences despite high overall sequence diversity at this locus among all samples analyzed. Such segments, if immunogenic, suggest the potential of a pregnancy-associated malaria vaccine based on a limited number of strains.

\section{Methods \\ Sample collection, genomic DNA preparation, and quantification of host contamination}

A total of 3-5 mL of venous whole blood were obtained from 12 children with symptomatic malaria episodes in Bandiagara, Mali, in 2010, as part of a study measuring the incidence of malaria at a vaccine testing site [22]. Whole blood samples were leukocyte-depleted at the time of collection using CF11 columns [23] and then kept frozen at $-80{ }^{\circ} \mathrm{C}$ until DNA extraction. DNA was extracted using the QIAamp Blood Midi kit, yielding $300 \mu \mathrm{L}$ of each sample eluted in distilled water. Samples were stored at $-80{ }^{\circ} \mathrm{C}$ until DNA sequencing. We determined clonality using six neutral microsatellite markers $[24,25]$. Host contamination was calculated retrospectively by measuring the proportion of sequenced reads that mapped to the human genome (version GRCh37) (see below). Isolate NF54 was provided by Sanaria, Working Cell Bank SAN02-073009, cultured using standard protocols [26], and DNA extracted as above.

\section{Generation of Illumina and PacBio whole-genome sequence data and genome assemblies}

Genomic DNA libraries for all samples were constructed for sequencing on the Illumina platform using the KAPA Library Preparation Kit (Kapa Biosystems, Woburn, MA, USA). First, $500 \mathrm{ng}$ of DNA was fragmented with the Covaris E210 to about $200 \mathrm{bp}$. Then libraries were prepared using a modified version of manufacturer's protocol. The DNA was purified between enzymatic reactions and the size selection of the library was performed with AMPure XT beads (Beckman Coulter Genomics, Danvers, MA, USA). The polymerase chain reaction (PCR) amplification step was performed with primers 
containing an index sequence six nucleotides in length. Libraries were assessed for concentration and fragment size using the DNA High Sensitivity Assay on the LabChip GX (Perkin Elmer, Waltham, MA, USA). The library concentrations were also assessed by quantitative PCR using the KAPA Library Quantification Kit (Complete, Universal) (Kapa Biosystems, Woburn, MA, USA). The libraries were pooled and sequenced on a $100 \mathrm{bp}$, paired-end, Illumina HiSeq 2500 run (Illumina, San Diego, CA, USA).

For the Malian clinical samples, DNA was prepared for PacBio sequencing using the DNA Template Prep Kit 2.0 (Pacific Biosciences, Menlo Park, CA, USA). First, DNA was fragmented with the Covaris E210 to generate fragments $\sim 3,8$, or $10 \mathrm{~kb}$ in length. Libraries were then prepared per the manufacturer's protocol. Three SMRT cells were sequenced per library, using P4C2 chemistry and a 120-min movie on the PacBio RS II (Pacific Biosystems, Menlo Park, CA, USA). The process for the generation of NF54 PacBio data was slightly different; NF54 had a longer insert library size $(18,800 \mathrm{bp})$ and was sequenced using P6-C4 chemistry.

Two assemblies were generated for each sample using PacBio-only sequencing data or PacBio plus Illumina data. PacBio-only assemblies were created with Sprai using default settings (http://zombie.cb.k.u-tokyo.ac.jp/ sprai/index.html); Sprai by default does not use reads shorter than 2000 bp. Hybrid assemblies using both PacBio and Illumina data were created with Celera assembler, with default settings [27].

\section{Extraction of var gene-like sequences from whole-genome assemblies}

Sequences resembling var exon 1 were extracted from the whole-genome assemblies by stitching together lenient amino acid-level alignments to known reference var genes. Amino acid alignments were generated using PROmer (promer -maxmatch) [28] between each wholegenome assembly and the full set of known exon 1 sequences from 3D7 and VarDom [13]). Matches of at least $50 \%$ identity at least $100 \mathrm{bp}$ long were grouped together when they were no more than $100 \mathrm{bp}$ apart, and any such group with a total length of at least $2000 \mathrm{bp}$ was considered a candidate exon 1 sequence. These candidate sequences were then oriented according to the reading frame with the least number of stop codons. Finally, duplicates and subsequences were eliminated by removing any sequence that was covered to at least $90 \%$ (proportion of length $\times$ proportion of identity) by another.

\section{Algorithm for var gene reconstruction using ETHA}

Likely ends (500 bp) of var exon 1 sequences were identified in each PacBio assembly by aligning known exon 1 ends from all var genes available in the VarDom database, including the var genes from the reference 3D7 genome, against each assembly, using NUCmer with option maxmatch [28]. Regions of the assembly from the start of each such match to $100 \mathrm{bp}$ past the inferred end of exon 1 were extracted from the assembly and then kmer-corrected, using all 71 mers observed at least ten times in the Illumina data. These repaired sequences were re-aligned to the reference exon 1 ends, the exact end identified as the rightmost AAGGT occurring in the rightmost $100 \mathrm{bp}$ of the match, and the $71 \mathrm{mer}$ ending with that AAGGT was added to the set of identified exon 1 end kmers. A similar approach was used for some other variants including ACCTT (the reverse complement of AAGGT) and other 5mers occurring with low frequency at the end of var exon 1 . This set of splice sitecontaining 71mers was then used to start a leftward kmer walk. This iteratively follows each $71 \mathrm{mer}$ with a new $71 \mathrm{mer}$ which overlaps the previous exactly over 70 shared base pairs, extending it by one base pair.

This process is followed iteratively until no overlapping kmers are found (with an occurrence count of at least ten) or a stop codon is encountered in all reading frames. Kmers at the left-most end of an open reading frame are collected as the starting point for a rightward walk following the same method, with the added stopping condition that a walk will end as soon as a kmer is seen which was in the original set of splice sitecontaining kmers. These rightward walks are used to identify further splice site kmers by finding the rightmost kmer ending in AAGGT, in each kmer walk terminated by the end of all reading frames. These new splice-site kmers are added to the original set and the leftward walk is repeated, this time using the seeds of the rightward walk as an additional stopping condition. All of the kmers encountered in this final leftward walk are saved as the set of possible 71mers belonging to exon 1. A final leftward walk is carried out to generate all possible sequences that start with the most recent set of splice-site kmers, using only the exon 171 mers. These sequences are filtered to remove any that is shorter than $1 \mathrm{~Kb}$ and the resulting set forms the candidate exon 1 sequences. These candidate exon 1 sequences are aligned to the whole-genome assembly and segments are extracted of at least $500 \mathrm{bp}$ aligning at least $90 \%$ identity. The extracted sequences are then assembled using a simple unitigger which requires perfectly matching overlaps.

We then identify all sequences formed by a unique path from the starting kmer and add them to the final set of exon 1 sequences if not already present. Duplicates, defined as sequences fully contained within any exon 1 unitigs, are removed. Also removed are sequences which are completely covered by another sequence with which they share $\geq 95 \%$ sequence identity. 


\section{NF54 validation}

An optimal NF54 PacBio-only assembly was constructed from all PacBio data using PacBio's Hierarchical Genome Assembly Process (HGAP) [29]. A second, sub-standard assembly that matched features of the 12 Malian clinical samples was generated from reads subsampled from the total NF54 PacBio data to match the lower median read length and read number of the datasets for Malian clinical samples. This simulated clinical assembly was created with HGAP using the subset of PacBio reads with the whitelist option within SMRT Analysis (v2.3). 71 mers used for kmer walk by ETHA were extracted from NF54 Illumina data. The original standard bioinformatic approach and ETHA were then run on this assembly to pull extracted and to generate reconstructed exon 1 sequences, respectively, as described above. For both extracted and reconstructed NF54 output, exon 1 sequences were then compared to the complete set of known exon 1 sequences from the reference 3D7 by alignment using Mummer [28] and coverage and percent identity was calculated for the single best match from the ETHA output to each reference 3D7 exon 1 sequence with custom scripts.

\section{Amplification and structural annotation of var2csa sequences from genomic DNA}

Primers flanking var2csa, based on its upstream promoter sequence (UPSE; 500 bp upstream of translation start site) and on the acidic terminal sequence (ATS), were designed using sequences from GenBank (accession numbers: EF614224; EF614233; EF614230; EF614227; EF614231; EF614225; EF614226; EF614229; EF614232; EF614228) and VarDom (clones 3D7, HB3, DD2, IT4/ FCR3, PFCLIN, RAJ116, IGH). UPSE sequences were provided by Thomas Lavstsen [13]. A single PCR was performed using PacBio 96-plex 21 bp padded barcode mode according to manufacturer's instructions. A target specific forward primer Frag1_ExF (5'-GTGATGTATGTGTTTA TGGAATAACTAGC-3') and a target specific reverse primer R_IT4var04_ATS (5'-TCCTTACGTTCCATATTCCA CACTTC-3') were used. The cycling conditions were as follows: a $50 \mu \mathrm{L}$ reaction containing $5 \mu \mathrm{L}$ of $10 \mathrm{X}$ LA PCR buffer, $8 \mu \mathrm{L}$ of dNTPs (2.5 mM each), $5 \mu \mathrm{L}$ of each the forward and reverse primer $(1 \mu \mathrm{M}), 0.5 \mu \mathrm{L}$ of TaKaRa LA Taq ( 5 units $/ \mu \mathrm{L}$ ), and $2 \mu \mathrm{L}$ of DNA sample. Cycling conditions included an initial denaturation step of $2 \mathrm{~min}$ at $94{ }^{\circ} \mathrm{C}$, followed by $10 \mathrm{~s}$ at $98{ }^{\circ} \mathrm{C}$ denaturation, $10 \mathrm{~min}$ annealing and elongation step at $60{ }^{\circ} \mathrm{C}$, with 35 cycles followed by a final elongation step of $10 \mathrm{~min}$ at $72{ }^{\circ} \mathrm{C}$. Successful PCR products of $10 \mathrm{~kb}$ from each sample were purified and pooled in equimolar concentrations. The pooled amplicons were sequenced using a Single Molecule Real Time (SMRT) RS II amplicon sequencing protocol using P6C4 chemistry, and assembled with SMRT analysis v2.3 ConsensusTools. The VarDom server was used for the var gene domain annotation, according to the default protocol parameters.

\section{Identification of upstream promoter sequence (UPS) for each var gene in the Malian samples}

Regions upstream from the translation start positions of var genes in our assemblies were extracted for analysis as follows. First, a set of possible exon 1 sequences was created from kmers in Illumina reads, as described above. Then, all PacBio reads were mapped to these sequences using NUCmer and the best match for each PacBio read extracted if it was at least $500 \mathrm{bp}$ long and matched the reconstructed exon 1 with at least $95 \%$ sequence identity. The extracted sequences were then assembled in a simple, greedy fashion to create a set of exon 1 unitigs. Those unitigs containing the starts of exon 1 sequences were matched using NUCmer against contigs in the final assembly. The final set of promoter regions consisted of the assembly regions upstream of the exon 1 start of each match (up to 2000 bp but as short as $900 \mathrm{bp}$ if the end of the contig was reached) plus the first $100 \mathrm{bp}$ of exon 1 coding sequence.

To identify the coordinates of each promoter sequence in those upstream regions and the UPS class to which each belonged, a BLAST search was done of known promoter sequences against the var gene upstream sequences. Each BLAST hit was then scored to determine the best hits. The scores were determined by adding the length, percent identity, and the bit score of the alignment (inversely proportional to e-value, and of the same order of magnitude and the other two parameters). This scoring takes into account the three factors that most impact the quality of the BLAST hit. The percent identity had little impact on the scores, since nearly all of the hits have a percent identity between $90 \%$ and $100 \%$. The coordinates for all the BLAST hits were inferred from the alignments. The best hits were defined by keeping only those hits within $5 \%$ of the score of the top hit for each upstream sequence. The $5 \%$ threshold represents a natural inflection point in the distribution of hit scores (scores tended to drop off after a few very highly scoring hits; alternatively, scores declined gradually, but represented redundant hits, i.e. alternative alignments of the same promoter to the same target region). All redundant hits were removed from the set of best hits, such that only unique best hits remained. Most often this resulted in only one remaining hit of a "known" promoter against the upstream region of a var, which defined the UPS class, and with the alignment coordinates defining the UPS location. In cases where more than one hit remained, it was a result of either: (1) partially overlapping hit coordinates from "known" promoters from the same class; or (2) perfectly overlapping hits, but from 
"known" promoters of different classes. In (1), the final UPS coordinates were defined by the reunion of all overlapping hits. In (2), the UPS coordinates were common to all hits and the var was assigned to a hybrid class defined by all subject "known" promoters. Finally, a small number of var sequences appear to have more than one non-overlapping upstream promoter, although these could represent assembly artifacts.

\section{Identification of domain cassettes DC8 and DC13}

DC8 and DC13 containing sequences were retrieved from VarDom server. Each DBL and CIDR domain was identified and aligned separately along with the known DC8 and DC13. Phylogenetic trees were inferred to identify sequences that clustered with either DC8 or DC13 domains. Sequences that contained both DBL $\alpha 2$ and CIDR $\alpha 1.1$ were defined as DC 8 whereas those with DBL $\alpha 1.7$ and CIDR $\alpha 1.4$ were DC13.

\section{Phylogenetic tree and recombination analysis}

Multiple sequence alignments were performed with AQUA [30], a program that optimizes multiple alignment using MAFFT and MUSCLE. A maximum likelihood tree was reconstructed with RAxML-PTHREADS (version 8.2.4) [31], based on the AQUA output. The gamma model of substitution with rate heterogeneity was used, together with AUTO option, to determine the matrix of substitution that best fits the data. JTT and VT were identified as the best-scoring amino acid substitution models. The phylogenetic tree was visualized with interactive Tree of Life (iTOL version 3). To determine whether var2csa sequences cluster regionally, a multiple alignment was performed using nucleotide sequences with MAFFT L-iNS-i default options followed by careful manual evaluation and editing when required. The alignment was used as an input for recombination analysis using RDP4 version 4.36 [32]. The program detected regions of recombination in the alignment. Therefore, a Neighbor-Net analysis was performed to reconstruct a phylogenetic network incorporating recombination events, using Splitstree version 4.14.4, based on uncorrected p-distances [33].

\section{Results and discussion}

\section{De novo whole-genome assemblies}

Genomic DNA (gDNA) was obtained from leukocytedepleted blood from each of 12 clinical malaria samples collected in Bandiagara, a town in Mali, West Africa. This set included samples with both low (5000-25,000 parasites $/ \mu \mathrm{L})$ and high $(>150,000$ parasites $/ \mu \mathrm{L})$ parasitemia, as well as monoclonal and polyclonal infections (Additional file 1: Table S1). Illumina and PacBio data were obtained for all samples (see "Methods"). The insert size of Illumina libraries was in the range of 316-346 bp
(Additional file 1: Table S2). The 12 samples were multiplexed in a single Illumina HiSeq2500 channel and data generated corresponded to $78 \mathrm{X}-141 \mathrm{X}$ coverage of the $P$. falciparum genome per sample. A $3 \mathrm{~Kb}, 8 \mathrm{~Kb}$, and/or $10 \mathrm{~Kb}$ PacBio insert library was built for each sample (Additional file 1: Table S2). The total PacBio data, generated through PacBio's Single Molecule Real Time (SMRT) sequencing, was equivalent to $8 \mathrm{X}-52 \mathrm{X}$ coverage, depending on the sample. The varying amount of data per sample depended on the percent host contamination, the variation associated with multiplexing samples in an Illumina run, the number of PacBio SMRT cells sequenced, and the variation inherent to each SMRT cell (Additional file 1: Tables S1 and S2).

Assemblies for the 12 Malian clinical samples were created using either Celera (PacBio with Illumina) or Sprai (PacBio-only) assemblers (see "Methods"). In each case, the assembly considered to be best was selected based on various combinations of assembly metrics, including number of contigs, cumulative length of assembly, length of largest contig, and N50 (the length of the largest contig in the subset containing the smallest 50\% of all contigs) (Additional file 1: Table S3). The P. falciparum assemblies generated from the six monoclonal infections were usually $<30 \mathrm{Mb}$ in length, and consisted of $\sim 450$ large contigs $(\geq 10 \mathrm{~Kb})$ of an average maximum size of $325 \mathrm{~Kb}$. These large contigs together had an average cumulative length of $22 \mathrm{Mb}$ ( $\sim 4 \%$ of the reference genome), corresponding to the core, unique regions of the genome (Fig. 1; Additional file 1: Table S3). The assemblies of the six polyclonal samples were all longer than $30 \mathrm{Mb}$, and the number of large contigs was $\sim 20 \%$ greater than those in monoclonal samples, while the number of small $(<10 \mathrm{~Kb})$ contigs from the polyclonal samples was roughly twice as big as those from the monoclonal samples, reflecting the presence of multiple allelic forms. All assemblies contained at least 500 small contigs of $2-10 \mathrm{~Kb}$. These small contigs map primarily to telomeric and sub-telomeric regions, suggesting that they correspond to fragments of repeats or the multigene families preferentially located in these regions (Fig. 1; Additional file 2).

The size of all assemblies was larger than the reference 3D7 P. falciparum genome. This is likely due to two primary factors. First, the relatively low amount of PacBio data that could be generated for each clinical sample were insufficient to correct all internal errors in the PacBio data. As a result, each assembly likely contains redundant contigs that could not be merged due to incorrectly reconstructed nucleotide positions (Fig. 1). Second, more than one clone was detected in half of these samples; therefore, some of the redundancy in the assembly is likely due to nucleotide-level differences in orthologous regions from multiple parasites. This may 


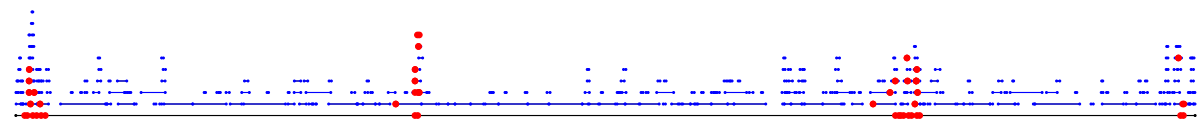

Chrm 12

Fig. 1 Whole-genome assembly contigs aligned against the 3D7 Reference. Contigs from Sample 303_1 (blue) aligned to chromosome 12 of 3D7 (black); var exon 1 sequences extracted from 303_1 (see "Methods") and previously identified var exon 1 sequences from 3D7 are labeled as red dots on the contigs and chromosome. Exon 1 sequences extracted from the whole-genome assembly of 303_1 are found in chromosome regions similar to where 3D7 exon 1 sequences have been found. While some are found towards the center of the chromosome, a portion are also found on the ends, where complicated repeat regions and multi-gene families cause assembly issues and assembly contigs are seen to pile up in this area. Similar layouts for all 14 chromosomes for each of the 12 Malian clinical samples can be found in the Additional files

explain why most assemblies from polyclonal infections are larger than those from monoclonal infections (Additional file 1: Table S3).

\section{Extracted var gene exon 1 sequences in de novo whole- genome assemblies}

Currently available $P$. falciparum genome assemblies contain 40-61 complete var genes in addition to several dozen var pseudogenes [34]. These genes are mostly located in sub-telomeric regions of the chromosomes, with a few clusters located in the core region of chromosomes 4, 6, 7, 8, and 12 (Fig. 1). Each complete var gene consists of two exons, the first of which, at 2500$10,500 \mathrm{bp}$, is longer and much more variable in length than the second (1000-1500 bp) [34]. While the PfEMP1 segment derived from exon 2 corresponds mostly to the inter-membrane and intra-cellular domains of the protein, exon 1 gives rise to the motifs involved in cytoadherence and rosetting, and consequently is of particular interest in terms of pathogenesis.

To determine the accuracy of these clinically important exon 1 sequences recovered directly from default assemblies with standard bioinformatics approaches, exon 1 sequences were extracted from each of the 12 Malian whole-genome assemblies based on amino acid sequence similarity to those of existing var exon 1 sequences from the reference $P$. falciparum 3D7 (Pf3D7) genome, as well as others available through the VarDom database [13]. The putative exon 1 sequences extracted were validated as bona fide var sequences by comparison with the NCBI $\mathrm{nr} /$ nt nucleotide collection. Without exception, each sequence was spanned (with or without internal gaps) by at least one match to a known var, with a bitscore of at least 200. Between 58 to 177 exon 1 sequences were extracted from each of the 12 Malian sample genome assemblies in this manner, with a higher number from polyclonal versus monoclonal samples (Additional file 1: Table S4). As with Pf3D7 var exon 1 sequences, the median length of extracted exon 1 sequences in each assembly was $\sim 5 \mathrm{~Kb}$ for most samples, and the length of individual exon 1 sequences fell within a wide range of 2 to $10 \mathrm{~Kb}$ (Fig. 2).

Several observations suggest the presence of assembly errors in the var exon 1 sequences extracted directly from the assemblies of the 12 Malian clinical samples. First, the number of exon 1 sequences per sample is much larger than the 40-61 expected. This is most likely due to the presence of duplicated segments in the assembly, which could not be merged due to incompatible sequencing errors (see above). Second, some segments in these extracted var sequences have no equivalent in the $k$-mer Illumina data, i.e. they have no identical sequence among all the 71 mers extracted from all Illumina reads generated for the same sample. Finally, in these extracted var sequences with missing $k$-mer data, no independently generated $k$-mer path (tiling paths of 71 mers extracted from all Illumina reads) unites the two regions flanking those segments with no $k$-mer coverage. The second observation can be explained by poor PacBio correction, but the final point suggests the presence of larger structural errors, such as chimeras. Therefore, the large number of complete exon 1 sequences extracted directly from these assemblies is inflated by the presence of chimeras, as well as by duplicated copies.

\section{Reconstruction of var gene exon 1 sequences by a novel algorithm, ETHA}

Compared to Pf3D7, the var exon 1 sequences extracted directly from each of the 12 genome assemblies were extremely variable in length and in number. In addition to the presence of duplicates and chimeras, the length distribution is skewed toward elements smaller than the median length, compared with Pf3D7. For this reason, an algorithm named ETHA was developed to more accurately recover exon 1 sequences (Fig. 3; see "Methods"). Briefly, Illumina data corresponding to the var genes is: (1) identified by finding 71 bp segments containing var splice site sequences in the assembly and iteratively following possible continuations within the Illumina data; (2) assembled by generating all possible paths within de Bruijn graph of 71mers; (3) reconciled with the whole-genome assembly by choosing paths that align best with the whole-genome assembly; and (4) assembling reconciled var sequences in a sample and removing duplicates. While the original extraction method described above was done using the best assemblies, we found that Celera assemblies (while often superior 

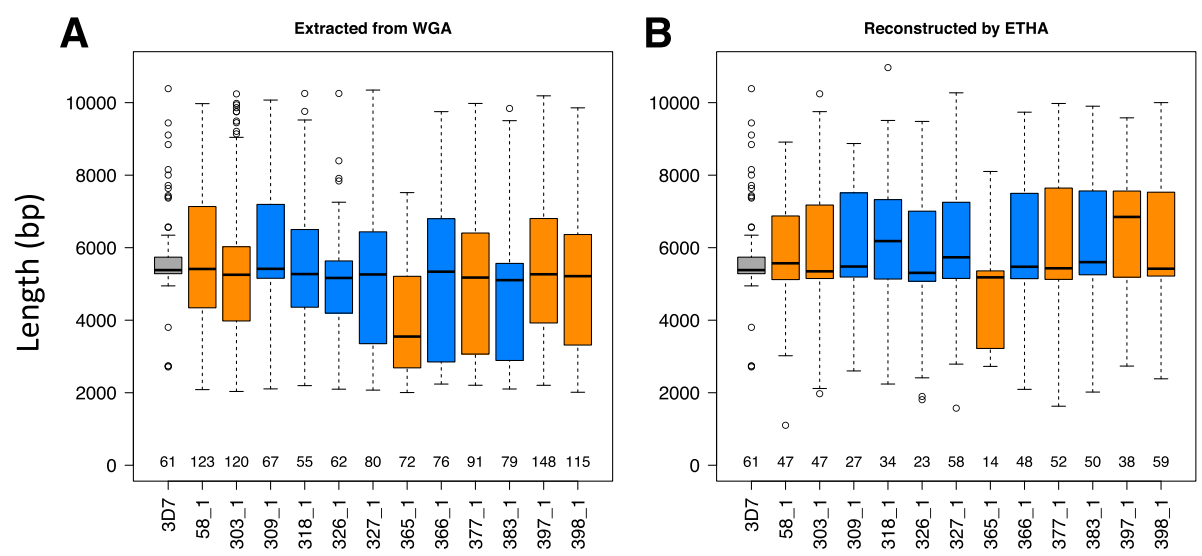

Fig. 2 Number and length of var exon 1 sequences. a Complete exon 1 sequence extracted from whole-genome assemblies using standard bioinformatic methods, requiring a minimum sequence length of $2 \mathrm{~Kb}$. $\mathbf{b}$ Complete exon 1 sequences reconstructed from whole-genome assemblies by ETHA. Distributions of var exon 1 lengths in each sample are represented by a box-and-whiskers plot: the median is indicated by a dark line, the first and third quartiles by the boundaries of the box, and the minimum and maximum, excluding outliers, by the whiskers. Outliers are those points lying beyond the first or third quartile by more than 1.5 times the interquartile range. Clinical samples are colored by clonality (polyclonal: orange, monoclonal: blue). 3D7 is shown in gray. The number of exon 1 sequences per sample, estimated from the counts of extracted exon 1 sequences are listed on the bottom (see "Methods")

as measured by N50 and other assembly metrics) were less effective than Sprai assemblies as input to the current pipeline, as measured by coverage of known reference var genes. Celera Assembler's double use of Illumina data may explain this reduced coverage.
Therefore, the Sprai assemblies were used as input for ETHA.

ETHA reconstructs both complete and partial exon 1 sequences. Those sequences containing a start methionine residue were judged to include the start of an exon;

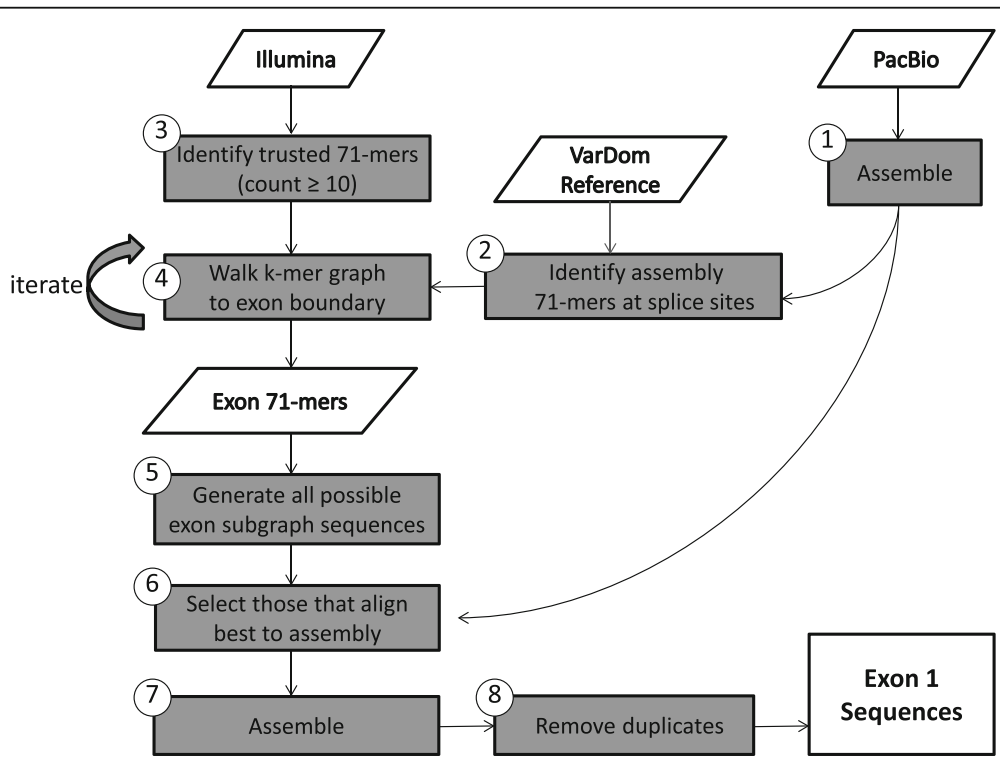

Fig. 3 Overview of the ETHA algorithm for reconstructing exon 1 sequences: Illumina and PacBio sequencing data are both used in conjunction with previously characterized exon 1 sequences from VarDom [13] as data inputs for reconstructing exon 1 sequences in clinical whole-genome assemblies. Pacbio data are assembled and exon 1 ends are identified by mapping known exon 1 sequences from VarDom onto the assembly (steps 1 and 2). Illumina data corresponding to var genes are identified by finding 71 bp segments (71mers) containing var splice site sequences at the end of exon 1 in the assembly and iteratively following possible continuations (new trusted 71 mers overlapping previously identified var 71 mers by $70 \mathrm{bp}$ ) within the Illumina data (steps 3 and 4). This process is extended until a start methionine is reached (step 4). This $\mathrm{k}$-mer walk is repeated in the opposite direction, now from the start methionine to the intron. They are then assembled by generating all possible paths within the de Bruijn graph of 71 mers (step 5) and reconciled with the whole-genome assembly by choosing those paths which align best with the whole-genome assembly (steps 6-8). Data inputs in white; processes are in gray. See "Methods" for additional details 
those ending with a known boundary sequence between exon 1 and the intron were judged to include an end. Those with neither were considered internal fragments and those with both were considered complete exon 1 sequences. The median length of complete ETHAreconstructed exon 1 sequences in each sample was much more similar to Pf3D7 and length variation was far lower than that of sequences extracted by the original method (Fig. 2; Additional file 1: Table S4). Since ETHA includes redundancy reduction steps that will remove nearly identical copies likely to result from sequencing error, the number of complete sequences reconstructed with ETHA is less than what was found with the exon 1 sequences extracted directly from the assemblies, and the total cumulative length of all ETHA exon 1 sequences was closer to what was expected (Additional file 1: Table S5). Finally, the estimated number of copies recovered by ETHA, which can be inferred from the sum of complete elements plus the largest of either the number of exon 1 start or end sequences (Additional file 1: Table S5), is also closer to number of complete elements in a genome than the number of var sequences extracted directly from each assembly.

\section{Validation of ETHA using NF54}

To confirm that ETHA is indeed recovering true and accurate var exon 1 sequences, we simulated the sequencing, assembly and var sequence recovery process used above for the 12 Malian clinical samples now using the P. falciparum isolate NF54. NF54 is the isolate from which the reference 3D7 was cloned [35]; therefore, any var exon 1 sequences present in NF54 should be very similar to those in the 3D7 genome. Indeed, this was confirmed with the generation of a high-quality PacBiobased whole-genome assembly of NF54, in which each of the $613 \mathrm{D} 7$ vars was present in one intact piece. We found no evidence in the NF54 assembly of extra var sequences relative to the original 3D7 assembly, as recently reported in a new PacBio-based 3D7 assembly [21]. The only difference detected between the NF54 and original 3D7 assemblies across the 61 genes was one small indel. The simulation was conducted by subsampling NF54 Illumina and PacBio reads such that the quality metrics for the data used to create the assembly and the ETHA pipeline input were on the low end of the Malian samples, in an attempt to replicate the relatively poor quality of the sequence data generated from the clinical samples (see "Methods") (Additional file 1: Table S3).

To assess sensitivity, specificity, accuracy, and contiguity of the ETHA algorithm, exon 1 sequences were extracted directly from the simulated NF54 assembly and also reconstructed with ETHA, as done before for the Malian clinical samples, and aligned to the known exon 1 sequences in the 3D7 reference. Overall, the ETHA output can be characterized as having very high accuracy and specificity at some cost to contiguity and sensitivity, while the direct extractions were quite sensitive and contiguous but at a cost to specificity and accuracy. ETHA output sequences covered $88.4 \%$ of the cumulative length of 3D7 var exon 1 sequences with alignments of at least $500 \mathrm{bp}$ and 100\% identity. Lowering the identity threshold to $90 \%$ identity increased the coverage only slightly to $92.8 \%$ and lowering it further to $80 \%$ achieved only $93.6 \%$ coverage. By contrast, the sequences extracted directly from the assembly covered only $17.2 \%$ of the cumulative length of the reference at $100 \%$ identity, but lowering the threshold to 90\% identity increased coverage to $97.3 \%$ and lowering it further to $80 \%$ achieved $97.8 \%$ coverage.

We also calculated coverage and percent identity for the single best match between the two sources. For the ETHA output, 38 of the 61 reference 3D7 exon 1 sequences were covered completely by a single match to reconstructed sequence, all of these with $100 \%$ identity. For the sequences extracted directly, only one reference exon 1 was covered completely with 100\% identity, but a total of 53 of the 61 were covered completely at lower identities (mean identity of $99.91 \%$, range of 99.58 99.98\%). The high number of complete (if not $100 \%$ accurate) exon 1 sequences extracted directly was most likely facilitated by the nature of the NF54 simulated sample that could not be degraded sufficiently to resemble true clinical data (likely due to the difference between clinical and cultured parasite genetic material and the longer reads from the updated PacBio chemistry used to sequence NF54; see "Methods"). The ETHA output's low number of completely covered reference 3D7 exon 1 sequences likely reflects ETHA's conservative nature, which by design will not merge two pieces of sequences unless they are identical across the overlapping region.

\section{Validation of ETHA using long-range targeted PCR with PacBio sequencing of var2csa}

A second validation of the ETHA output was done by sequencing var2csa with PacBio to compare the PCR amplicon-based sequence to the sequence reconstructed by ETHA. PCR primers spanning the UPS and ATS were used to amplify the extracellular portion of var2csa, corresponding to an amplicon around $10 \mathrm{~Kb}$, and then sequence it with PacBio using P6-C4 chemistry (see "Methods"). For all except two samples (397_1 and 398_1), the var2csa gene was successfully amplified and sequenced. The ten amplicons were pooled before sequencing with PacBio.

We assembled the PacBio reads with PacBio's HGAP assembler and further corrected the results using Pilon, with the corresponding Illumina reads [36]. Three of the ten samples (309_1, 366_1, and 383_1) had very low 
PCR amplicon product and did not yield the desired amount (394.94 ng) to generate an equimolar tensample amplicon pool before sequencing. Therefore, the number of PacBio reads for these samples was insufficient to generate a reliable PacBio consensus amplicon sequence. Of the remaining samples, two (318_1 and 377_1) failed to yield high-quality assemblies; for the last five samples, we obtained a single high-confidence contig assembly, thus providing an independent source against which to assess the accuracy of the ETHA pipeline. In four of these five samples (303_1, 326_1, 327_1, and 365_1), the assembled amplicon sequence corresponding to exon 1 was $100 \%$ identical, end to end, to the output of the ETHA pipeline, while the fifth (58_1) differed by 11 nucleotides from the ETHA output, corresponding to $99.87 \%$ identity. In each of the five samples, the most similar var2csa-like sequence extracted directly from the whole-genome assembly differed considerably from the assembled amplicon, with differences in the range of 6-2750 nucleotides (median $=287 \mathrm{bp}$ ). This validation exercise provides further evidence that ETHA produces sequences of higher accuracy than those obtained using standard bioinformatic approaches to extract sequences directly from a default, low coverage PacBio assembly.

As a positive control for this validation approach, we also amplified and PacBio-sequenced the exon 1 of var2csa from the 3D7 isolate. The sequence obtained was $100 \%$ identical to that in the published 3D7 genome assembly.

\section{Subfamily composition}

The upstream regions of var genes have previously been classified into four UPS classes, namely UPSA, UPSB, UPSC, and UPSE, which correspond to various chromosomal locations and clinical phenotypes [13]. UPSA (associated with longer var genes) and UPSB (the most common) tend to be sub-telomeric, whereas UPSC is located centrally on chromosomes. UPSE is associated only with var2csa. To determine if the var genes reconstructed from the 12 clinical samples shared a similar promoter breakdown, we extracted 2100 bp-long segments upstream of each exon 1 found in the wholegenome assemblies of the 12 Malian samples, and classified these by sequence similarity to the four UPS classes (see "Methods"). As has been observed in other genomes, UPSB was the most common UPS class for each of the clinical samples (Fig. 4). While previous work found a similar number of UPSA and UPSC classes in a parasite genome [13], UPSA tended to be more numerous than UPSC in the 12 Malian clinical isolates studied here. As expected, UPSE-like sequences were present at very low numbers in each sample. Five samples had between two to four UPSE copies, half of the samples had a single UPSE copy, and none were found in sample 318_1. In contrast, sample 377_1 had 15 UPSE-like sequences. The large number of UPSE sequences relative to the four var2csa genes reconstructed with ETHA from the 377_1 assembly likely stems from redundancy in these low coverage PacBio assemblies and sample polyclonality.

\section{Structural characterization of var2csa and other vars using VarDom}

All ETHA exon 1 sequences were characterized and annotated using the VarDom server ([13]; www.cbs.dtu.dk/ services/VarDom/) (see "Methods"). Briefly, the exon 1 genomic sequences were used to predict open reading frames (ORF); the resulting ORFs were translated into protein sequences and submitted to VarDom for domain prediction. The output files were parsed with custom Python and Perl scripts to extract domain coordinates and determine protein domain organization. The overall domain organization was consistent between the samples (Fig. 5; Additional file 3: Figure S1), and similar to the var composition in the 3D7 reference genome, with NTSDBL $\alpha$-CIDR $\alpha-D B L \delta-C I D R \beta$ being the most frequent arrangement and NTS-DBL $\alpha-C I D R \alpha-D B L-C I D R \gamma$ the second most common domain architecture. While this domain architecture was conserved, individual PfEMP1 sequences were highly polymorphic. The most conserved vars from the repertoire were var1, var3, and var2csa. According to the structural characterization of all samples, var1, var3, and var2csa variants were present in nine, seven, and 12 samples, respectively. Complete or nearly complete var $2 c s a$ exon 1 sequences were recovered from all 12 samples, with some samples having more than one var2csa sequence, providing cross-validation of the output of the ETHA algorithm. Each sample possessed a similar distribution frequency of constituent domains in these three most widespread vars as the 3D7 isolate. The most common domain found in each sequence was DBL $\alpha$ (Additional file 1: Table S6).

PfEMP1s play an important role in malaria pathogenesis by mediating the binding of infected erythrocytes to specific host receptors in different organs. Particular clinical features have been associated with specific domains or the combination of two or more domains defined as domain cassettes (DC). In particular, the expression of DC8 and DC13 has been associated with severe malaria [37]. Previously, DC8 was found in six of seven genomes from different continents, while DC13 was found in five of these genomes [13]. The reference 3D7 genome lacks DC8. To identify these domain cassettes in our dataset, we reconstructed phylogenetic trees using amino acid sequence of domains from ETHA-reconstructed exon 1 sequences as well as amino acid sequences of known DC13 and DC8 domain cassettes. Both domain cassettes 


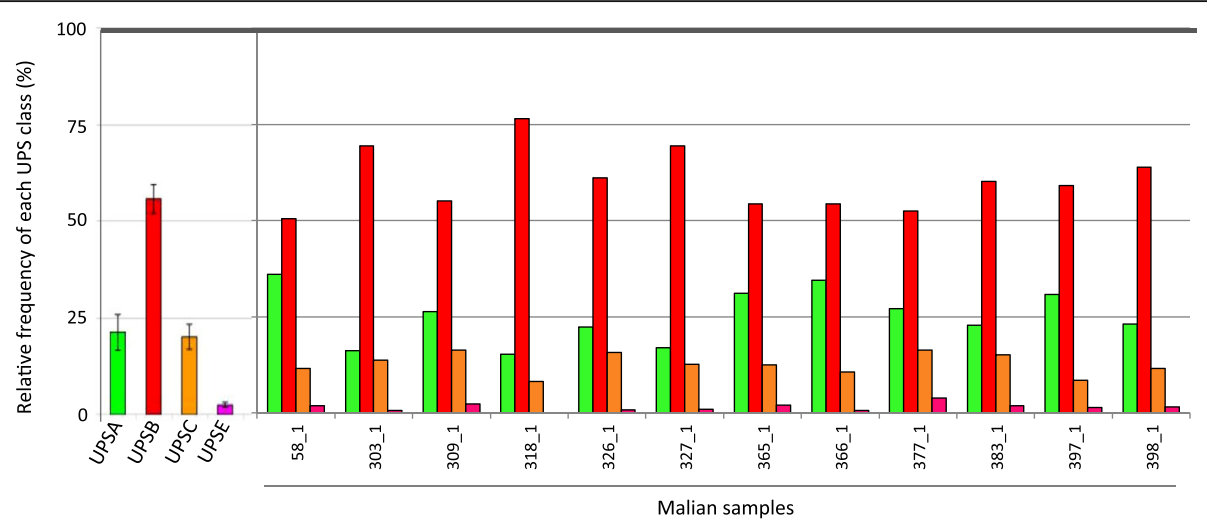

Fig. 4 Subfamily composition of recovered ETHA exon 1 sequences: relative frequencies of each UPS class (UPSB in red, UPSA in green, UPSC in orange, and UPSE in purple) for each of the 12 Malian samples, compared to the frequencies found by Rask et al. [13]

were identified in 11 samples at least once (Additional file 3: Figures S2 and S3). Samples 58_1 and 397_1 had more than one DC8 (paired domains). We detected neither DC8 nor DC13 in sample 318. These results suggest that differences in clinical outcome may be the result of variation in var expression and/or host immunity, instead of being directly related to domain composition of the var complement in the genome. These findings may also be explained by other factors such as the timing of sample collection, the possibility that the DC8s and DC13s sequenced in these samples differ, in some critical way, from DC8s and DC13s that have been associated with severe malaria, or that these uncomplicated malaria infections could have progressed to severe infections if left untreated.

\section{Genetic diversity of var2csa and other vars}

To characterize the diversity of var repertoires, average amino acid identity was calculated for each domain of the major DBL and CIDR classes, as well as the entire exon 1 region of var2csa. Among a sample of vars obtained from a collection of isolates from around the globe, amino acid sequence identity of individual domains was in the range of $30-92 \%$ [13]. The DBLe (31\% shared amino acid identity) and CIDR $\alpha$ domain (30\% shared amino acid identity) classes were the most heterogeneous domains, whereas DBL $\beta$ and CIDR $\delta$ were relatively more conserved, with $43 \%$ and $55 \%$ shared amino acid identity, respectively (Additional file 1: Table S7). Remarkably, both the relative sequence conservation among domains and the range of amino acid sequence identity observed among 12 Malian samples are essentially identical to global isolates (Additional file 1: Table S7). We also confirmed that VAR1 and VAR3 were among the most conserved vars, with $67 \%$ and $87 \%$ shared amino acid identity, respectively. The high level of conservation of these two proteins among parasites suggests particular-and as yet undiscovered-niches in malaria pathogenesis that warrant further investigation.

The genetic variation among VAR2CSA proteins is particularly pertinent to the development of a pregnancyassociated malaria vaccine. We identified and characterized the genetic diversity among the 19 different var2csa sequences found in the 12 Malian samples. The average shared amino acid sequence identity among these sequences was $79 \%$. Sequence identity within the individual

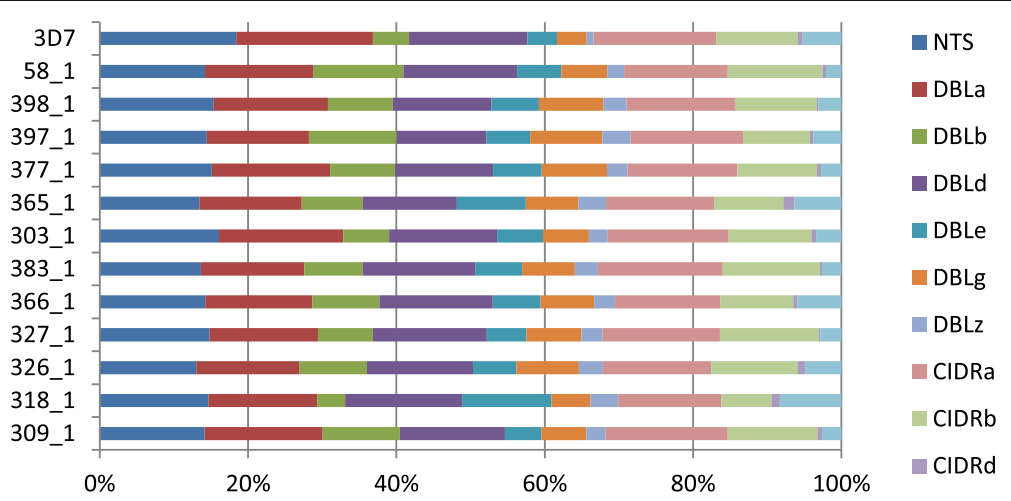

Fig. 5 Relative distribution of constitutive domains. Relative domain frequencies compared to the reference 3D7 
constituent domains ranged from 62\% (DBLE10) to 92\% (DBLepam4). DBLepam4 was the most homogeneous domain, followed by DBLepam5 and DBLpam3, both with $89 \%$ amino acid sequence identity (Additional file 1: Table S7). The ID1-ID2a region of the VAR2CSA protein, which contains the motif that binds the CSA binding region, is the target of current efforts to develop a vaccine against malaria in pregnancy. The average amino acid sequence identity for this region was $74 \%$, suggesting that it has considerable heterogeneity even among parasites from a single West African village. In addition, all 19 Malian ID1-ID2a sequences were unique.

To determine the relatedness among var2csa sequences from parasites collected in different geographic locations, Malian sequences were aligned and compared to var2csa sequences retrieved from the VarDom database, which were obtained from parasites from across the globe. A principal component analysis revealed that var2csa sequences from Mali did not form a distinct cluster; instead, Malian samples displayed a large range of genetic diversity in the principal component analysis (PCA) (Fig. 6a). Supporting this conclusion, phylogenetic analyses showed Malian var2csa sequences not to be monophyletic; in fact, several Malian sequences were more closely related to var2csas from other continents than they were to other Malian samples (Fig. 6b). The phylogenetic network was reconstructed to account for recombination, since recombination events were detected in the data, both among Malian samples and between these and other samples (Additional file 3: Figure S4). An analysis under the assumption of no recombination differed slightly in topology but still showed Malian samples to be polyphyletic in the network (Additional file 3: Figure S5). These results, taken together, suggest that VAR2CSA sequences are not structured according to geography, supporting the idea that a VAR2CSA-based vaccine based on a number of strains within a region would still capture considerable global var2csa sequence diversity.

Despite the tremendous variation among var2csa sequences from a single Malian village, two VAR2CSA C-terminal extracellular domains, DBLepam4 and DBLepam5, were remarkably conserved among these isolates. In a recent study using the 3D7 VAR2CSA variant [38], sera from multigravid women from Mali recognized a fragment containing these two domains to a greater extent than sera from nulliparous women, men, and children. Other studies have also shown that these domains were targets of maternal antibodies [39-41]. Our results suggest that the high sequence conservation of the extracellular C-terminal makes it a potential target for a subunit vaccine. Immune responses and allele-specific immunogenicity against different variants of these domains are important to characterize, as previous studies suggest that a repertoire of antibodies from women recognize both conserved and polymorphic epitopes [42].

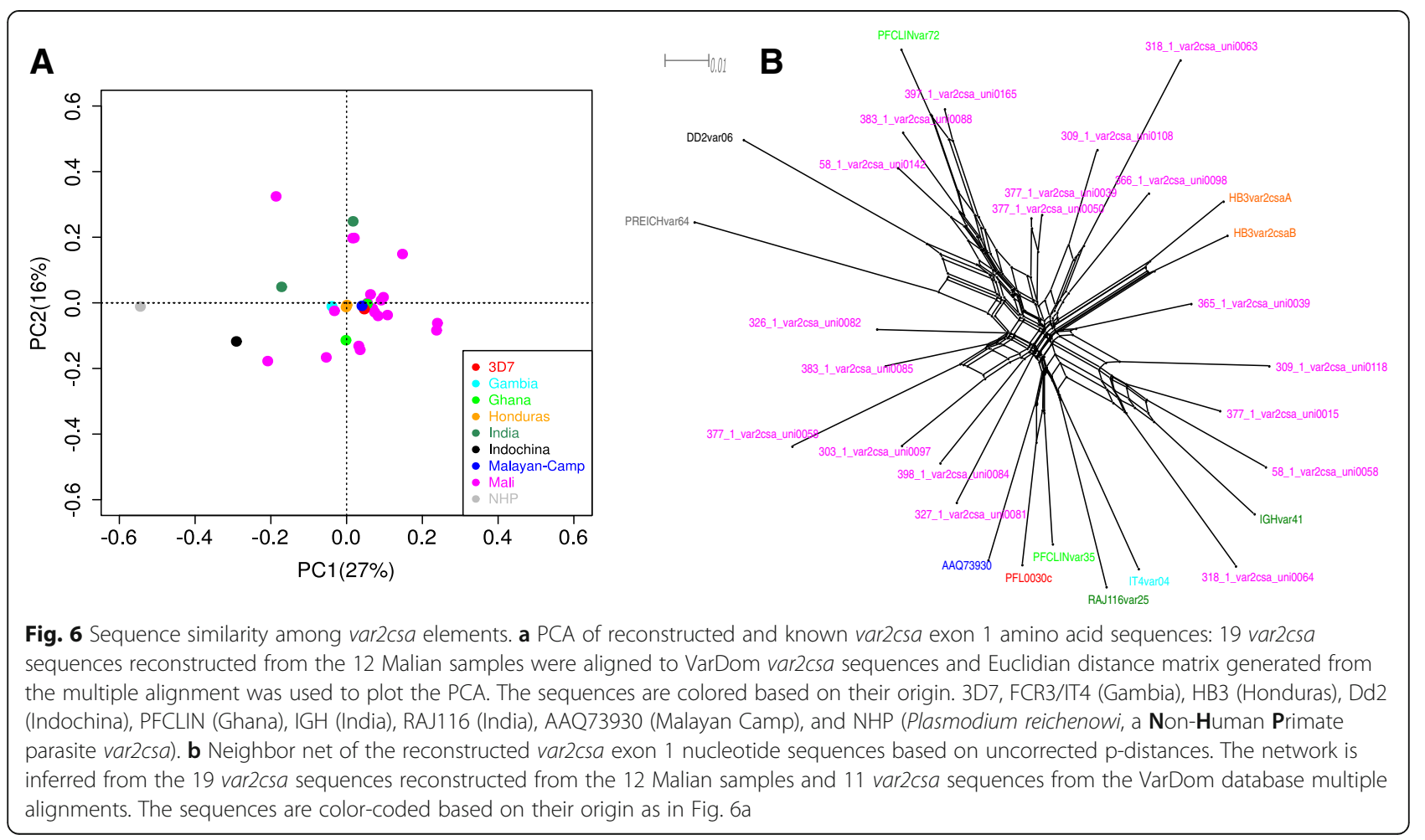




\section{Conclusions}

Sequence analysis of PfEMP1s from clinical isolates has the potential to provide insights into malaria pathogenesis and vaccine design. Challenges in sequencing var genes from clinical isolates have limited our understanding of P. falciparum pathogenesis and ability to develop interventions focused on this family of variant proteins. We overcame these challenges by combining two sequencing technologies and developing and validating a novel assembly algorithm. PacBio offered the advantage of long reads, permitting assembly of vars, while Illumina corrected the inherent sequencing errors associated with PacBio. ETHA is a novel algorithm for reconstructing variable var exon 1 sequences from unfinished, highly fragmented whole-genome assemblies generated from clinical samples using PacBio and Illumina data. This approach allowed us to accurately and reproducibly reconstruct the sequence of full-length $\mathrm{var}$ exon 1 from clinical isolates, in an automated fashion, for the first time. While var sequences extracted directly from the assemblies likely encompass almost all var exon 1 sequences present in the sample, collection of sequences was both chimeric and redundant, and contained sequencing errors. In contrast, ETHA removed much of this duplication and reduced chimerism and sequencing errors, albeit with a slight cost in sensitivity and contiguity, resulting in some fragmentation. The accuracy of ETHA var reconstruction was validated using several approaches, including the recovery of known vars and the validation of the motif composition of novel reconstructed vars.

This approach will provide valuable var sequence information for clinical infections, with the potential to vastly expand the limited available sequence data from the field, with associated clinical and epidemiological data. The pipeline described here can be adapted to other surface variant proteins such as those encoded by the repetitive interspersed family (rif) and the sub-telomeric variable open reading frame (stevor) gene families, which have yet to be sequenced from clinical infections.

This approach has some limitations. Sequence generation is dependent on the acquisition of adequate quantities of venous blood (typically $3-5 \mathrm{~mL}$ ) with a sufficient parasitemia to guarantee enough parasite DNA for at least a small insert PacBio library-here, a minimum of 5000 parasites per microliter. In addition, the cost of necessary next-generation sequencing may be prohibitive for some projects. Finally, these blood samples required leukocyte depletion in the field to reduce host DNA contamination. New developments to deplete human DNA from mixed host-parasite DNA samples, coupled with sequencing technology advances to reduce costs and improve sensitivity may soon overcome some of these limitations.
Comparative sequence analysis showed that the repertoire of var genes found in a small number of isolates from a single West African village was as variable as a collection of isolates from different continents. However, two immunogenic VAR2CSA C-terminal regions of exon 1 [38-40] exhibited considerable sequence conservation, suggesting a potential target for vaccine development. Additional var sequence data from different geographic settings would serve to accurately determine epitope region variability. Such information would allow better representation of PfEMP1 diversity on protein and peptide microarrays used to assay malaria exposure and immunity [38, 43-45].

We have shown that parasite sequences present in uncomplicated malaria infections included var2csas and also var subtypes associated with severe malaria. Severity of clinical infection was thus not dictated by the potential for virulence of the var repertoire present in an infection. The presence of DC8 and DC13 in the genomes of parasites in uncomplicated malaria infections contradicts a hypothesis that severe malaria is caused by highly restricted, virulent strains of $P$. falciparum [46], even though some caveats to this point remain, as mentioned in "Results." Additional studies of host immunity, specific var gene regulation and expression, and var gene subsets in different clinical syndromes are needed to better understand determinants of virulence.

\section{Additional files}

\footnotetext{
Additional file 1: A file containing Supplementary Tables S1-S7. Table S1: Samples and respective metadata. Table S2: Genomic DNA: Illumina and PacBio Sequencing Statistics. Table S3: Assembly characteristics for 12 Malian samples generated by Sprai and Celera assemblers. Table S4: Exon 1 var sequences recovered with ETHA compared to raw var-like sequences extracted directly from assemblies. Table S5: Percent of extracted sequence absent from the ETHA output. Table S6: Comparison of PfEMP1 constitutive domains reconstructed from 12 samples to the reference 3D7. Table S7: Average percent (\%) amino acid identity within PfEMP1 constitutive domains. (DOCX $64 \mathrm{~kb}$ )
}

Additional file 2: Contig pile-ups of the genome of $12 P$. falciparum isolates from Mali aligned against the reference 3D7 genome. The genome assembly of each of the 12 isolates, represented by its constituent contigs (blue lines in between blue circles) is aligned against each of the 14 nuclear chromosomes of the reference $P$. falciparum 3D7 strain (black). The location of var gene sequences is shown (red) in both the 3D7 genome and the contigs aligned to it. (PDF $1608 \mathrm{~kb}$ )

Additional file 3: A file containing Supplementary Figures S1-S5. Figure S1: Domain organization of PfEMP1 in each of 12 Malian isolates. Figure S2: Maximum likelihood phylogeny of DBLa domain sequences. Figure S3: Maximum likelihood phylogeny of CIDRa domain sequences. Figure S4: Detection and visualization of recombination events within and between var2csa sequences. Figure S5: Phylogenetic tree of reconstructed var2csa exon 1 sequences. (DOCX $3594 \mathrm{~kb}$ )

\section{Abbreviations}

ATS: Acidic terminal sequence; CIDR: Cysteine-rich interdomain region; DBL: Duffy-binding-like; DC: Domain cassettes; ETHA: Exon-Targeted Hybrid Assembly; HGAP: Hierarchical Genome Assembly Process; ORF: Open reading frame; PacBio: Pacific Biosciences; PfEMP1: Plasmodium falciparum erythrocyte 
membrane protein-1; SMRT: Single Molecule Real Time; UPS: Upstream promoter sequence

\section{Acknowledgements}

We thank Thomas Lavstsen for providing the VarDom var2csa upstream regions (UPSE). We also thank Danzele Coulibaly, Sekouba Mariko, Moctar Traore, Nicole Eddington Johnson, and Amanda Icenroad for administrative support; Matthew Adams, Sandra Mon, and Andrea A. Berry for laboratory support; the team of the Bandiagara Malaria Project in Bandiagara for their dedication; and the community of Bandiagara, Mali.

\section{Funding}

This work was supported by a cooperative agreement (U19Al065683) from the National Institute of Allergy and Infectious Diseases (NIAID), National Institutes of Health (NIH) (CVP); NIAID/NIH GSCID Contract \# HHSN272200900009C (CMF, JCS, CVP, MAT); NIAID/NIH U19AI1 10820 (CMF, JCS, CVP); NIH grant D43TW001589 from the Fogarty International Center to AD; a Burroughs Wellcome Fund/ American Society of Tropical Medicine and Hygiene Postdoctoral Fellowship to MAT, and an award to CVP from the Howard Hughes Medical Institute. The funding bodies had no role in the design of the study, in collection, analysis, and interpretation of data, or in writing the manuscript.

\section{Availability of data and material}

Sequencing data are available through NCBI's Short Read Archive (SRA) and can be located with the following sample accession numbers: SAMN02373653, SAMN02373656, SAMN02373818, SAMN02373819, SAMN02373820, SAMN02373821, SAMN02373822, SAMN02374900, SAMN02374901, SAMN02374902, SAMN02374907, SAMN02374945, and SAMN06175781. The source code for ETHA is available at: https://sourceforge.net/projects/etha/.

\section{Authors' contributions}

JCS, MAT, AD, and CVP conceived and designed the study. JCS and MAT oversaw the study. EFD and ALD designed and developed the ETHA algorithm. QS assembled the sequence data. AD, EFD, KAM, and TH tested and validated ETHA. AD conceived and designed the amplicon validation approach and performed experiments. MAT, DC, MD, AD, ID, AKK, BK, AN, KT, YT, MAT, AAD, OKD, CVP, and CMF contributed materials and analysis tools. JCS, AD, EFD, MAT, $\mathrm{KAM}, \mathrm{MBL}$, and CVP wrote the manuscript. All authors read and approved the final manuscript.

\section{Competing interests}

The authors declare that they have no competing interests.

\section{Consent for publication}

Participants consented to publication of study results provided that they were only identified by participant number.

\section{Ethics approval and consent to participate}

This study uses Plasmodium falciparum DNA from blood samples collected from participants in a study of malaria infection and disease incidence at a malaria vaccine testing site in Bandiagara, Mali. Patients were consented, through their parents or legal guardians, to participate in the study. The Ethics Committee of the Faculty of Medicine, Pharmacy and Odonto-Stomatology of the University of Bamako (FWA00001769) and the University of Maryland Institutional Review Board (FWA00007145) approved the research protocols (FMPOS approval No. 09 38, DMID Protocol Number: 08-0022, UMB IRB Protocol Number: HP-00041382).

\section{Publisher's Note}

Springer Nature remains neutral with regard to jurisdictional claims in published maps and institutional affiliations.

\section{Author details}

'Division of Malaria Research, Institute for Global Health, University of Maryland School of Medicine, Baltimore, MD, USA. ${ }^{2}$ Institute for Genome Sciences, University of Maryland School of Medicine, Baltimore, MD, USA. ${ }^{3}$ Malaria Research and Training Center, University of Science, Techniques and Technologies, Bamako, Mali. ${ }^{4}$ Department of Microbiology and Immunology, University of Maryland School of Medicine, Baltimore, MD, USA. ${ }^{5}$ Department of Medicine, University of Maryland School of Medicine, Baltimore, MD, USA.
Received: 20 September 2016 Accepted: 2 March 2017 Published online: 28 March 2017

\section{References}

1. Crompton PD, Kayala MA, Traore B, Kayentao K, Ongoiba A, Weiss GE, et al. A prospective analysis of the $\mathrm{Ab}$ response to Plasmodium falciparum before and after a malaria season by protein microarray. Proc Natl Acad Sci U S A. 2010;107:6958-63.

2. Marsh K, Otoo L, Hayes RJ, Carson DC, Greenwood BM. Antibodies to blood stage antigens of Plasmodium falciparum in rural Gambians and their relation to protection against infection. Trans R Soc Trop Med Hyg. 1989;83:293-303.

3. Oh SS, Voigt S, Fisher D, Yi SJ, LeRoy PJ, Derick LH, et al. Plasmodium falciparum erythrocyte membrane protein 1 is anchored to the actin-spectrin junction and knob-associated histidine-rich protein in the erythrocyte skeleton. Mol Biochem Parasitol. 2000;108:237-47.

4. Baruch DI, Pasloske BL, Singh HB, Bi X, Ma XC, Feldman M, et al. Cloning the $P$. falciparum gene encoding PfEMP1, a malarial variant antigen and adherence receptor on the surface of parasitized human erythrocytes. Cell. 1995;82:77-87.

5. Newbold Cl, Craig AG, Kyes S, Berendt AR, Snow RW, Peshu N, et al. PfEMP1, polymorphism and pathogenesis. Ann Trop Med Parasitol. 1997;91:551-7.

6. Rogerson SJ, Chaiyaroj SC, Ng K, Reeder JC, Brown GV. Chondroitin sulfate A is a cell surface receptor for Plasmodium falciparum-infected erythrocytes. J Exp Med. 1995;182:15-20.

7. Treutiger CJ, Heddini A, Fernandez V, Muller WA, Wahlgren M. PECAM-1/ CD31, an endothelial receptor for binding Plasmodium falciparum-infected erythrocytes. Nat Med. 1997:3:1405-8.

8. Rowe JA, Claessens A, Corrigan RA, Arman M. Adhesion of Plasmodium falciparum-infected erythrocytes to human cells: molecular mechanisms and therapeutic implications. Expert Rev Mol Med. 2009;11:e16.

9. Scherf A, Lopez-Rubio JJ, Riviere L. Antigenic variation in Plasmodium falciparum. Annu Rev Microbiol. 2008;62:445-70.

10. Kyes $S$, Horrocks $P$, Newbold C. Antigenic variation at the infected red cell surface in malaria. Annu Rev Microbiol. 2001:55:673-707.

11. Smith JD, Gamain B, Baruch DI, Kyes S. Decoding the language of var genes and Plasmodium falciparum sequestration. Trends Parasitol. 2001:17:538-45.

12. Kraemer SM, Kyes SA, Aggarwal G, Springer AL, Nelson SO, Christodoulou Z, et al. Patterns of gene recombination shape var gene repertoires in Plasmodium falciparum: comparisons of geographically diverse isolates. BMC Genomics. 2007:8:45.

13. Rask TS, Hansen DA, Theander TG, Gorm Pedersen A, Lavstsen T. Plasmodium falciparum erythrocyte membrane protein 1 diversity in seven genomesdivide and conquer. PLoS Comput Biol. 2010;6:e1000933.

14. Jespersen JS, Wang CW, Mkumbaye SI, Minja DT, Petersen B, Turner L, et al. Plasmodium falciparum var genes expressed in children with severe malaria encode CIDRalpha1 domains. EMBO Mol Med. 2016;8:839-50.

15. Fried M, Nosten F, Brockman A, Brabin BJ, Duffy PE. Maternal antibodies block malaria. Nature. 1998:395:851-2.

16. Salanti A, Dahlback M, Turner L, Nielsen MA, Barfod L, Magistrado P, et al. Evidence for the involvement of VAR2CSA in pregnancy-associated malaria. Jxp Med. 2004:200:1197-203.

17. Bockhorst J, Lu F, Janes JH, Keebler J, Gamain B, Awadalla P, et al. Structural polymorphism and diversifying selection on the pregnancy malaria vaccine candidate VAR2CSA. Mol Biochem Parasitol. 2007;155:103-12.

18. Doritchamou J, Sabbagh A, Jespersen JS, Renard E, Salanti A, Nielsen MA, et al. Identification of a major dimorphic region in the functionally critical N-Terminal ID1 domain of VAR2CSA. PLoS One. 2015;10:e0137695.

19. Manske M, Miotto O, Campino S, Auburn S, Almagro-Garcia J, Maslen G, et al. Analysis of Plasmodium falciparum diversity in natural infections by deep sequencing. Nature. 2012;487:375-9.

20. Chien JT, Pakala SB, Geraldo JA, Lapp SA, Humphrey JC, Barnwell JW, et al. High-quality genome assembly and annotation for Plasmodium coatneyi, generated using Single-Molecule Real-Time PacBio technology. Genome Announc. 2016:4:e00883-16.

21. Vembar SS, Seetin M, Lambert C, Nattestad M, Schatz MC, Baybayan P, et al. Complete telomere-to-telomere de novo assembly of the Plasmodium falciparum genome through long-read (>11 kb), single molecule, real-time sequencing. DNA Res. 2016:23:339-51.

22. Coulibaly D, Travassos MA, Kone AK, Tolo Y, Laurens MB, Traore K, et al. Stable malaria incidence despite scaling up control strategies in a malaria vaccine-testing site in Mali. Malar J. 2014;13:374. 
23. Venkatesan M, Amaratunga C, Campino S, Auburn S, Koch O, Lim P, et al. Using CF11 cellulose columns to inexpensively and effectively remove human DNA from Plasmodium falciparum-infected whole blood samples. Malar J. 2012;11:41.

24. Shaukat AM, Gilliams EA, Kenefic $\sqcup$, Laurens MB, Dzinjalamala FK, Nyirenda $\mathrm{OM}$, et al. Clinical manifestations of new versus recrudescent malaria infections following anti-malarial drug treatment. Malar J. 2012;11:207.

25. Anderson TJ, Su XZ, Bockarie M, Lagog M, Day KP. Twelve microsatellite markers for characterization of Plasmodium falciparum from finger-prick blood samples. Parasitology. 1999;119(Pt 2):113-25.

26. Trager $W$, Jensen JB. Human malaria parasites in continuous culture. Science. 1976;193:673-5.

27. Myers EW, Sutton GG, Delcher AL, Dew IM, Fasulo DP, Flanigan MJ, et al. A whole-genome assembly of Drosophila. Science. 2000;287:2196-204.

28. Delcher AL, Phillippy A, Carlton J, Salzberg SL. Fast algorithms for large-scale genome alignment and comparison. Nucleic Acids Res. 2002;30:2478-83.

29. Chin CS, Alexander DH, Marks P, Klammer AA, Drake J, Heiner C, et al. Nonhybrid, finished microbial genome assemblies from long-read SMRT sequencing data. Nat Methods. 2013;10:563-9.

30. Muller J, Creevey CJ, Thompson JD, Arendt D, Bork P. AQUA: automated quality improvement for multiple sequence alignments. Bioinformatics. 2010;26:263-5.

31. Stamatakis A. RAxML version 8: a tool for phylogenetic analysis and postanalysis of large phylogenies. Bioinformatics. 2014;30:1312-3.

32. Martin DP, Lemey P, Lott M, Moulton V, Posada D, Lefeuvre P. RDP3: a flexible and fast computer program for analyzing recombination. Bioinformatics. 2010;26:2462-3.

33. Huson DH, Bryant D. Application of phylogenetic networks in evolutionary studies. Mol Biol Evol. 2006;23:254-67.

34. Gardner MJ, Hall N, Fung E, White O, Berriman M, Hyman RW, et al. Genome sequence of the human malaria parasite Plasmodium falciparum. Nature. 2002;419:498-511.

35. Walliker D, Quakyi IA, Wellems TE, McCutchan TF, Szarfman A, London WT, et al. Genetic analysis of the human malaria parasite Plasmodium falciparum. Science. 1987;236:1661-6.

36. Walker BJ, Abeel T, Shea T, Priest M, Abouelliel A, Sakthikumar S, et al. Pilon: an integrated tool for comprehensive microbial variant detection and genome assembly improvement. PLoS One. 2014;9:e112963.

37. Lavstsen T, Turner L, Saguti F, Magistrado P, Rask TS, Jespersen JS, et al. Plasmodium falciparum erythrocyte membrane protein 1 domain cassettes 8 and 13 are associated with severe malaria in children. Proc Natl Acad Sci U S A. 2012;109:E1791-1800.

38. Travassos MA, Coulibaly D, Bailey JA, Niangaly A, Adams M, Nyunt MM, et al. Differential recognition of terminal extracellular Plasmodium falciparum VAR2CSA domains by sera from multigravid, malaria-exposed Malian women. Am J Trop Med Hyg. 2015;92:1190-4.

39. Barfod L, Bernasconi NL, Dahlbäck M, Jarrossay D, Andersen PH, Salanti A, et al. Human pregnancy-associated malaria-specific B cells target polymorphic, conformational epitopes in VAR2CSA. Mol Microbiol. 2007;63:335-47.

40. Tutterrow YL, Avril M, Singh K, Long CA, Leke RJ, Sama G, et al. High levels of antibodies to multiple domains and strains of VAR2CSA correlate with the absence of placental malaria in Cameroonian women living in an area of high Plasmodium falciparum transmission. Infect Immun. 2012;80:1479-90.

41. Oleinikov AV, Rossnagle E, Francis S, Mutabingwa TK, Fried M, Duffy PE. Effects of sex, parity, and sequence variation on seroreactivity to candidate pregnancy malaria vaccine antigens. J Infect Dis. 2007;196:155-64.

42. Beeson JG, Mann EJ, Byrne TJ, Caragounis A, Elliott SR, Brown GV, et al. Antigenic differences and conservation among placental Plasmodium falciparum-infected erythrocytes and acquisition of variant-specific and cross-reactive antibodies. J Infect Dis. 2006;193:721-30.

43. Travassos MA, Niangaly A, Bailey JA, Ouattara A, Coulibaly D, Laurens MB, et al. Seroreactivity to Plasmodium falciparum erythrocyte membrane protein 1 intracellular domain in malaria-exposed children and adults. J Infect Dis. 2013;208:1514-9.

44. Barry AE, Trieu A, Fowkes FJ, Pablo J, Kalantari-Dehaghi M, Jasinskas A, et al. The stability and complexity of antibody responses to the major surface antigen of Plasmodium falciparum are associated with age in a malaria endemic area. Mol Cell Proteomics. 2011;10:M111.008326.

45. Dent AE, Nakajima R, Liang L, Baum E, Moormann AM, Sumba PO, et al. Plasmodium falciparum protein microarray antibody profiles correlate with protection from symptomatic malaria in Kenya. J Infect Dis. 2015;212:1429-38.

46. Gupta S, Hill AV, Kwiatkowski D, Greenwood AM, Greenwood BM, Day KP. Parasite virulence and disease patterns in Plasmodium falciparum malaria. Proc Natl Acad Sci U S A. 1994;91:3715-9.

\section{Submit your next manuscript to BioMed Central and we will help you at every step:}

- We accept pre-submission inquiries

- Our selector tool helps you to find the most relevant journal

- We provide round the clock customer support

- Convenient online submission

- Thorough peer review

- Inclusion in PubMed and all major indexing services

- Maximum visibility for your research

Submit your manuscript at www.biomedcentral.com/submit 